\title{
Automatic Assessment of Student Understanding Level using Virtual Reality
}

\author{
Shota Hashimura \\ Graduate School of Information Science and Engineering \\ Ritsumeikan University, Shiga, Japan \\ Email: hashimura@de.is.ritsumei.ac.jp
}

\author{
Hiromitsu Shimakawa, Yusuke Kajiwara \\ College of Information Science and Engineering \\ Ritsumeikan University, Shiga, Japan \\ Email: \{simakawa, kajiwara\}@de.is.ritsumei.ac.jp
}

\begin{abstract}
The improvement of the efficiency in teaching requires knowing the understanding level of each student. However, it is difficult due to limited time in a class. We propose a Virtual Reality (VR) space imposing assignments on students, to know their understanding level from their behavior which comes from cognitive loads during their answering. The VR space presents a student an assignment and a working space to answer it. In general, students solve assignments, using elements on their short term memory. When students solve same kind of assignments many times, they build generalized solution methods in their long term memory. When they engage in such assignments, their cognitive load is low enough to make them watch only the working spaces, keeping their hands working. On the other hand, when students have no solution pattern, their short term memory works hard. Their high cognitive load often stop their hands, because of confusion. They also look assignments and the working space many times, to reconsider solutions. Since answering behavior of students exposes their cognitive load, a VR space is ideal to estimate cognitive load. We conducted an experiment to evaluate the ability of the method to estimate the cognitive load. We examined the movement of the hand and the edit distance of student's answer from the correct sentence during their answering. We confirmed a fair correlation of the hands' stagnation with the confidence in students of good scores. We also found a relationship of eye movement with the change of the edit distance. The experiment result implies the possibility to estimate the cognitive load. The estimation would enable teachers to know students' understanding faults, which leads to education according to the understanding level.
\end{abstract}

\section{INTRODUCTION}

I $\mathrm{N}$ EDUCATION, adjusting the difficulty of tasks in the class maximizes the learning effect[1][2]. Students cannot learn anything from too difficult tasks, nor anything from too easy tasks. Therefore, teachers try to adjust the progress of classes. For the adjustment, they need to know the cognitive load on students. In the context, the cognitive load in learning refers to the total amount of mental activity imposed on students. In face to face class, teaches can estimate the load, looking their behaviors. Teachers can also ask questions to students, to receive feedbacks from students. Such direct communications is the best way to know the cognitive load on students, but it takes so long time to communicate with large number of students. To avoid it, teachers assign students paper tests or e-learning tests instead of direct communication. Although these tests can check understanding level of many students at one time, teachers cannot know behavior of students from these tests. These tests cause miss-understanding of the cognitive load. One example is a correct answer by luck. In addition, it is a hard work for students and teachers to perform tests many times. It is troublesome to adjust the difficulty level of the class for students. We must find the easy way to estimate student understanding level correctly.

There are two types of memories in human brain: a working memory and a long term memory. Each of the memories has its own functions. We focus on difference of these functions to estimate understanding level. The function of the working memory is information processing to understand situations and carry out tasks. Its capacity is limited[4] and the memory is lost within about $20 \mathrm{~s}[5]$. On the other hand, the long term memory has large size, to store patterns which are often used in the processing in the working memory. Each of the patterns is treated as one chunk, when it is restored from the long term memory to the working memory. The patterns are referred to as schemata[6].

To solve tasks which are not mastered well, students need to process information without schema. It is hard for students, because the working memory should store a lot of information at the same time[7]. Since the capacity of the working memory is small, the tasks make the cognitive load high. By contrast, when students master the tasks through repeated practices, they restore the schemata corresponding to them from the long term memory to the working memory. Since schemata combine several pieces of information into one chunk, they help students reduce the number of pieces on the working memory. Consequently, schemata reduce the cognitive load[8][9][10].

When students make mistakes and show hesitation in learning tasks, they seem not to have established schemata on the knowledge to achieve the tasks. They seem to have high cognitive load caused by a lot of information on the working memory. Based on the idea, this study proposes a method to estimate student understanding level correctly from their behaviors to answer tasks in learning using a VR space. In this study, we utilize tests to sort English words in a VR space. In a VR space, we can record detailed behavior such as gaze shifts and hand movements. We analyze the behavior along with test results, to estimate their cognitive load. The estimation reveals student understanding level. In addition, this paper discusses a way to examine what part of the learning task imposes the 
high cognitive load on the students, which enables us to find what knowledge they lack.

In this paper, section II explains the relationship of operation in a VR space with cognitive load. Section III clarifies the method to figure out the understanding level from behavior. Section IV presents an asessment system of understanding level using a VR tool. Section V evaluates the method by an experiment. Section VI discusses the result of the experiment. Section VII concludes our works.

\section{RELATIONSHIP OF OPERATION IN VR SPACE WITH COGNITIVE LOAD}

\section{A. Cognitive load}

When people understand matters, their brains memorize the information on the matter. It imposes the loads on their brain. The load is referred to as cognitive load. People cannot understand the matter without memorizing it. Some people take things as they are. Other people connect the related things as single facts before they remember them. It is good to group the related facts as single ones, in order to reduce the load of the memories. People would memorize the pattern of related things which are often used on the memories. The patterns of related things and processing results are treated as chunks[11]

There are two types of human memory: the working memory and the long term memory. Each memory is specialized for their role.

The working memory stores the information temporarily to process it. People must store all information on tasks in their working memory to achieve them[2][3]. Nevertheless, the capacity of the working memory is small[4]. It is reported the working memory only can store around four pieces of information even in the case of young adults[12].

By contrast, the long term memory has large capacity. The long term memory stores the pattern of thinking and relationship of information. It is referred to as a schema. A schema can combine several chunks as a bigger chunk. The combination makes the load of the working memory smaller[8][9][10]. For example, let us assume to remember a sequence of six letters of "MEMORY". If a child who does not know English tries to remember this alphabet sequence, the child has to memorize each character like ' $M$ ', ' $E$ ', 'M', 'O', 'R', 'Y'. On the other hand, if you know English word "MEMORY", you can combine that information as one chunk, which reduces the burden on the working memory. The difficulty of a learning task depends on the cognitive load, while the cognitive load is determined by knowledge of students. Students who have appropriate knowledge to solve questions can decrease the number of chunks in the working memory. Therefore, the cognitive load is also reduced.

Students can learn no knowledge from too difficult assignments, because they cannot proceed the task. In the same way, too easy assignments give no knowledges to students, because there are no new things for them. Estimation of the cognitive load can change the teaching, because we can adjust the difficulty for each student to maximize the effect of learning.

\section{B. Human sense in VR space}

In VR space, the movement of users is measured to make the users feel they move in the space, as if they move in the real space. The movement is measured with 2 wearable devices: a head mount display, and handy mortion controllers.

The head mount display, which is used to display the VR space to users, measures the position and the rotation of the user, to display the virtual space naturally to the user. The space presented inside the head mound display changes according to the head movement, so that the users take their views just like in their ordinal life. They can see anything in a VR space from any position and any direction in the way they want. For this reasons, users can take three important factors to feel reality, 3D spatiality, real time responsibility and self-projecting[13].

Recent VR can detect hands movement using handy motion controllers. Due to the motion controllers, users can interact with VR objects. We can know quickly the detailed position and rotation of the motion controllers. We can also detect the grasping of users. Therefore, we can reproduce their hands in VR space, through the projection of the virtual hand models on the position where the users feel their real hands are placed. In addition, the device enables us not only to rotate the virtual hands as the actual hands rotate, but also to bend the virtual fingers as the real fingers. Since the movement of virtual hands is identical with real ones, the reality is provided wiht users.

Suppose students engage in learning tasks in in a VR classroom. Using wearable devices, we can detect the movement of the gaze and the hand of the students. Users can interact with virtual objects without operative difficulties, because they can grasp virtual objects as they do in the real life. The records expressing their behaviors contain few noises. The movement shows their hesitation, confidence and cognitive load in the learning tasks. It shows purely their understanding level.

\section{Related works}

Many studies try educational data mining[14]. For example, Ivancevic, Celikovic \& Lukovic find the seats selection of students in classroom, which is related to their assessment[15].

There are some studies to reveal the understanding level of e-learning students. As one example, Nakamura[16] used a camera to analyze the facial movement. His team succeeded estimating $75 \%$ of the subjective difficulty of the students from their facial behavior. However, facial behavior depends on individuals. The method requires a specialized estimator for each student. It has also a problem to record the behavior of the students by a camera, from the viewpoint of privacy.

Eye gaze is used to know focus and attention of users[17]. In the case of VR, eye gaze is one of the pointing way to interact virtual objects without hand interaction. Some of head-mount displays can detect gaze of users(e.g. FOVE[18]). However, they are expensive. Since most of them cannot detect the gaze, they use head-based interactions as a proxy of gaze pointing[19][20]. This pointing is an operation, which is a conscious behavior. To estimate the students understanding, it 
is also important to analyze the unconscious behavior such as taking a look at hints.

We need a method to estimate each student understanding level. To do this, we should find predictors which explain the understanding level of individual students.

\section{CLARIFYING UNDERSTANDING LEVEL FROM BEHAVIOR}

\section{A. Recording behavior consisting of small tasks}

We propose the method which estimates student understanding level from their behavior. A task of a specific student consists of small tasks. When the student finishes each of the small tasks, the main task is over too. We focus on these small tasks such as looking at a question and picking up a word card. We record the order of these small tasks as behavior, which is the target data to be analyzed.

After teachers estimate the understanding level from behavior, they can improve their classes with the records so that the classes suit for the student understanding.(Fig. 1)

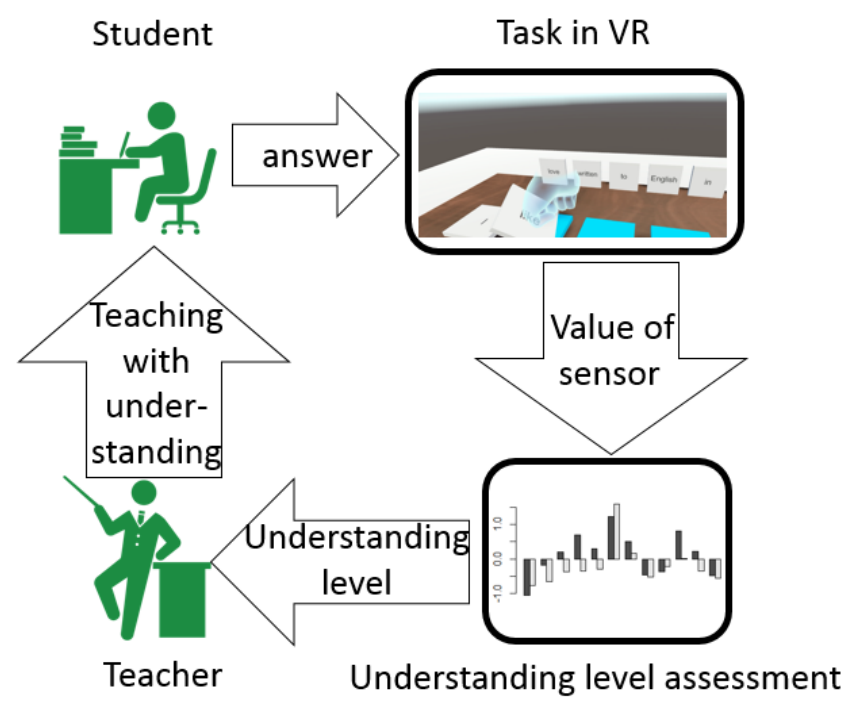

Fig. 1. Operation of proposal method

\section{B. Estimating understanding level of each student}

This paper takes an example which is a sort test of English words to explain the way to assess student understanding level. The tests are conducted in a VR space. Every student who tries the test wears a head mount display and hand gears specific to the VR space. The pair of the head mount display and the hand gears enables the student to experience the sort test provided in the VR space. At the same time, the pair is equipped functions to record the movement of the head and the both hands of the student.

We record movement of the hands and the head of the student in VR test (Fig. 2). When students have low cognitive load, the movement of their hands and head is smooth. On the other hand, when students have high cognitive load, the movement often stops. Our research estimates the cognitive

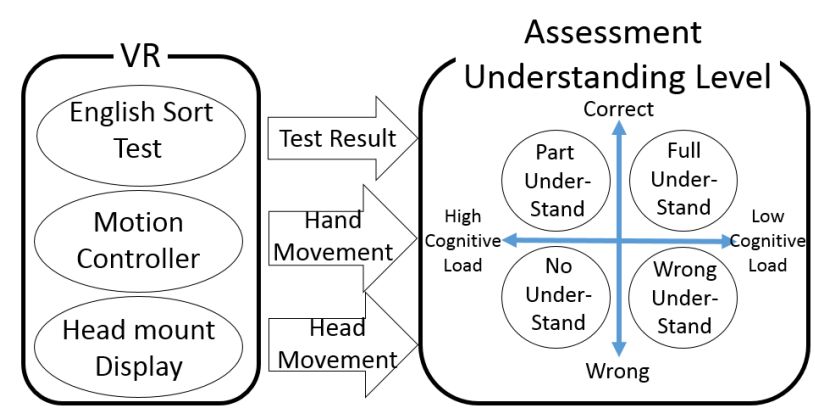

Fig. 2. Method overview

load from the movement. Furthermore, we reveal what students do not understand through analysis of test results and the cognitive load, which is estimated from their behavior.

\section{Assessment of cognitive load}

We can estimate the student understanding level with the analysis of the cognitive load, deeper than that without behavior. We quantify the cognitive load based on machine learning. Since cognitive load is phenomenon in brain, it is not observable. It is difficult to quantify. However, students who solve questions with low cognitive load have high confidence, while high cognitive load makes confidence low. We can know the cognitive load from confidence of students appearing on their behavior. We examine the following two relationships to assess the cognitive load.

(1) Relationship between the hand movement and the confidence

As we mentioned, high confidence is assumed to make hands movement smooth. We tried to confirm this assumption.

(2) Relationship between the gaze movement and the edit distance

If students have high confidence, they do not look same place repeatedly. This gaze movement seems to be related to edit distance, which represents the number of how many correction is needed to finish the English sort test. In the test, we assume the more the edit distance is, the higher the cognitive load gets, because it is more difficult for the students to image correct sentences. To make the assumption confirmed, we examine the relationship between the gaze movement and the edit distance.

\section{ASSESSMENT OF UNDERSTANDING LEVEL USING VR}

\section{A. VR English sort test}

We prepared a VR English sort test to know what behavior students show while they engage is the test. This test is constructed using Unity5.6.2 p2. Fig. 3 shows a snapshot of a participant sight when a student takes the test. The movie of experiment is located at www.de.is.ritsumei.ac.jp/publication/englishtest.mp4 


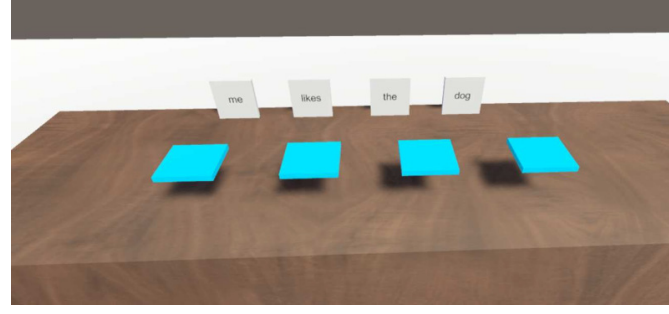

Fig. 3. Sight of students in VR test

In the experiment, participants move the words to correct order of sentence. The blue squares are columns for answer. Participants grab word cards by their hands to place them on those columns. As a hint, a Japanese sentence is presented above the word cards. The participants should look up the hint. When they look up and look around, the head mounted display they wear sense its position and direction. The data show the participant gaze movement which include the information of their understanding level.

\section{B. Head movement and gaze movement}

We used Oculus Rift, a head mount display. The participants engage in the test, wearing it on their heads. They usually look at a specific item in the VR space, to move the item from one place to another. For the participants, this behavior in the VR space is more natural than their behavior while they engage in conventional e-learning using a mouse to scroll pages. This head movement is related to the gaze movement. It implies the difficulty the participants have. Since students who have enough knowledge can imagine the correct English sentence consisting of the given words, they can solve the test without looking the hint many times. On the other hand, students fail to imagine the correct one try to make their answers, looking the hint many times and gazing many word cards repeatedly.

\section{Hand movement}

We used Oculus Touch, the motion controller. When users wear a pair of the motion controllers on their hands, virtual hands appear in VR space. The virtual hands move in the VR space according to that of the hands of users. Using the motion controllers, the users can grab and put things in the VR space by their hands. Since the movement of the virtual hands is coincident with that of the user hands, the VR test is better than conventional e-learning in terms of the ease of control. Because of the ease, the hand movement which students take while VR test contain information which achieves clear analysis of the student understanding level.

\section{EXPERIMENT}

\section{A. Experiment contents and purposes}

We experiment on the confidence of participants for a sort test of English words in the VR space.

There are two purposes in this experiment. One is to confirm the relationship between the movement of hands and the confidence. The other purpose is to examine the possibility to assess the confidence from their gaze movement.

In the experiments, 11 college students took assignments to sort English words in the VR space. We conducted 2 kinds of experiment. The first one provides 12 assignments taken by four students. The second one provides 15 assignments taken by 7 students. Some assignments are difficult, while others are easy. We recorded the movement of their heads and right hands every 20 milliseconds. Every time the students finish assignments, we asked their confidence for their answer. In the first experiment, they evaluate their confidence on a scale of 1 to 5 . In the second one, they used 4 grade evaluation. No students took both assignments.

\section{B. Relationship of hand movement with confidence}

We calculated the ratio of the time in which the hand is stopped to the whole answering time, where the stop of the hand means the sum of the absolute values of the hand location change in $\mathrm{x}, \mathrm{y}$, and $\mathrm{z}$ directions in 20 milliseconds is less than 2 millimeters. We examined the correlation between the ratio and the confidence.

Unfortunately, we experienced data missing for 2 students. Excluding the two student data, we analyzed the correlation of 9 students. The results are shown in Table I.

In the results, the correlation coefficient goes below -0.7 for seven of the nine students. It means $78 \%$ students have low confidence when they hold their hand in assignments. We confirmed the correlation coefficient between their confidence and the hand movement, which means we can estimate the confidence of students from their hand movements.

The two students who were low in the correlation got poor scores in the assignments than others. These students may have answered in the assignments without deep considerations.

\section{Relationship between gaze movement and edit distance}

Students who have low confidence to solve the assignments would repeat to look at the hints and the word cards alternatively. They are likely to show many gaze shifts which come from the alternative looking. On the other hand, students who have enough knowledge can imagine the correct sentences. They can solve the test without such an unnecessary gaze shift. Unnecessary gaze shifts of students seem to be related their indecision. We detected the amount of unnecessary gaze shifts by the method explained in Fig 4.

During the assignment, every student would look at the hint to determine a specific card word to grasp. Every 20 milliseconds, the method identifies the direction the head faces. It is calculated from the rotation of the head mount display. The method also figures out the vector which corresponds to the direction to the word card the student grabbed next. This vector starts from the student head, and reaches to the word card. The method calculates the cosine similarity of two vectors. When the direction of the two vectors is identical, the cosine similarity takes 1.0 , the highest value. It decreases, as the student gazes items located in other direction than that of the word card. We calculated the amount of unnecessary 
TABLE I

RELATIONSHIP OF HAND MOVEMENT WITH CONFIDENCE

\begin{tabular}{|c|c|c|}
\hline Students & Correlation Coefficient & Number of correct answers \\
\hline A & -0.806 & $8 / 12$ \\
\hline B & -0.91 & $8 / 12$ \\
\hline C & -0.911 & $7 / 12$ \\
\hline D & -0.568 & $5 / 12$ \\
\hline E & -0.897 & $11 / 15$ \\
\hline F & -0.704 & $10 / 15$ \\
\hline G & -0.845 & $9 / 15$ \\
\hline H & -0.765 & $6 / 15$ \\
\hline I & -0.380 & $5 / 15$ \\
\hline Mean & -0.754 & \\
\hline
\end{tabular}
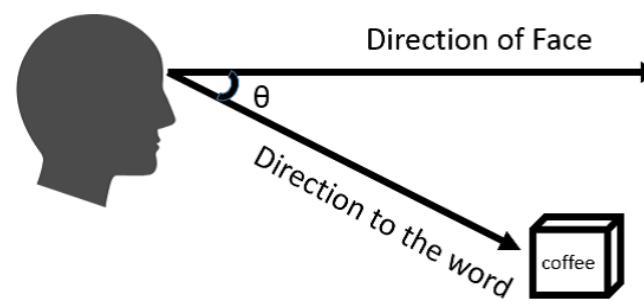

$$
\Delta t=\left\{\begin{array}{cl}
\cos \theta_{t}-\cos \theta_{t-1}, & , \cos \theta_{t}<\cos \theta_{t-1}-0.01 \\
0 & , \cos \theta_{t} \geq \cos \theta_{t-1}-0.01 \\
\text { Unnecessary gaze shift } \mathrm{V}=\sum_{t} \Delta t
\end{array}\right.
$$

Fig. 4. How to detect a unnecessary gaze shift

gaze shifts as the sum of decline of the cosine similarity from the grasp of one word card to another. We ignore the difference of the cosine similarity more than -0.01 during 20 milliseconds, because it means the student gaze the same item in the duration.

When students lack enough knowledge, they would modify the word order in their answers many times, because they have poor confidence. The frequent modification increases the edit distance of their answers against the correct answers. We tried the multiple regression analysis, where the edit distance is the response variable, while the explanatory variables are the amount of gaze shifts and the time for the students use to answer. The result is showed in Table II. All students except the student E have higher P-value than 0.05. Moreover, the adjusted R-squared coefficients are very low. It means there are no relationship the gaze shift and the edit distances.

After the experiment above, we reconsider the estimation model. We review processes in which students answer assignments. Putting words one by one, students would make sentences. In the process, students would look at not only placed words but also pre-placed words the students are required to be placed in the right order. Therefore, when we estimate the cognitive load, we should consider all words, though we addressed only placed words in the previous experiment.

We re-calculate the edit distance with all words including the placed words and pre-placed words(Fig. 5). We tried the multiple regression analysis to fit this new edit distance with

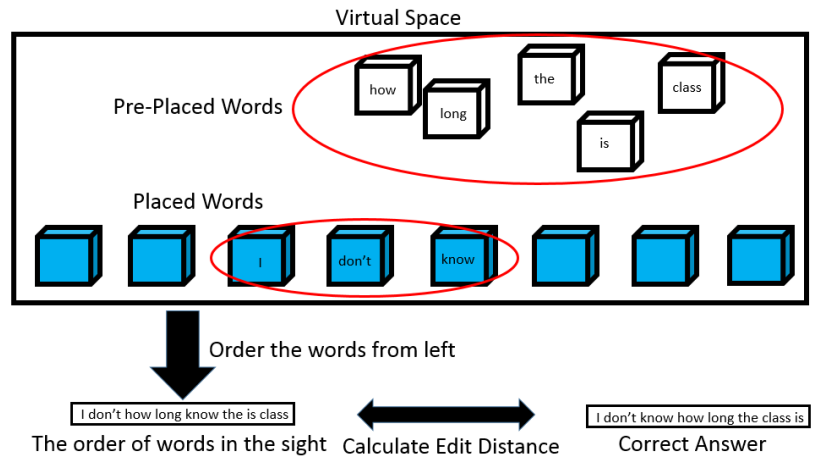

Fig. 5. Calculation of edit distance between correct answer and order of words in sight

the amount of gaze shifts and the answer time. The result is showed in Table III. The P-value is improved to below 0.05 for about 5 of 7 students. Moreover, the adjusted R-squared coefficient is also improved, although its average is still around 0.04 .

\section{DISCUSSION}

\section{A. Assessment the confidence from the behavior}

The negative correlation became weaker in two of nine students. They answered fewer correct answers. After the test, we interviewed these students. This interview revealed that one of them is likely to express low confidence even for assignments he quickly gave correct answers. The student may lose confidence than usual, because of successive high-level assignments. However, the other student often expresses high confidence even for wrong answers. The mismatches of the confidence from the answers seem to be caused by lack of knowledge. That is called Dunning-Kruger effect[21]. When the test is too difficult for them, they cannot understand what is correct, which makes them misunderstand the difficulty of questions. Our method cannot estimate the confidence whose scores are low. However, we can find the confidence of students who scored well. In other words, we can choose students suitable to estimate their confidence from their scores.

The adjusted-R squared was around 0.04 in the result of multivariate regression to fit the edit distance of all words, 
TABLE II

RESULT OF MULTIPLE REGRESSION ANALYSIS

\begin{tabular}{|l|l|l|l|l|l|}
\hline \multirow{2}{*}{ students } & \multicolumn{2}{|l|}{ Unnecessary Gaze Shift } & \multicolumn{2}{|c|}{ Thinking time } & \multirow{2}{*}{ Adjusted R-squared } \\
\cline { 2 - 5 } & Coefficient & P-value & Coefficient & P-value & \\
\hline E & 0.09769 & 0.025 & 0.10198 & 0.019 & 0.03112 \\
\hline F & -0.01961 & 0.667 & 0.06203 & 0.174 & 0.00739 \\
\hline G & -0.00121 & 0.977 & -0.0591 & 0.163 & 0.00331 \\
\hline H & -0.00632 & 0.855 & 0.00354 & 0.918 & -0.00585 \\
\hline I & 0.04637 & 0.364 & -0.00804 & 0.875 & -0.00427 \\
\hline J & 0.07278 & 0.099 & 0.08668 & 0.05 & 0.01497 \\
\hline K & -0.00632 & 0.855 & 0.00354 & 0.918 & -0.00585 \\
\hline Mean & 0.02292 & 0.48 & 0.02382 & 0.389 & 0.0051 \\
\hline
\end{tabular}

TABLE III

RESULT OF MULTIPLE REGRESSION ANALYSIS BY CONSIDERING ALL WORDS

\begin{tabular}{|l|l|l|l|l|l|}
\hline \multirow{2}{*}{ Students } & Unnecessary Gaze Shift & \multicolumn{2}{c|}{ Thinking Time } & \multirow{2}{*}{ Adjusted R-squared } \\
\cline { 2 - 5 } & Coefficient & P-value & Coefficient & P-value & \\
\hline E & 2.5853 & 0.00003 & 0.7614 & 0.21 & 0.08793 \\
\hline F & 1.7009 & 0.022027 & 1.2457 & 0.0922 & 0.02405 \\
\hline G & 0.9263 & 0.117 & 0.3426 & 0.561 & 0.002795 \\
\hline H & 0.6490 & 0.1487 & 0.4752 & 0.2900 & 0.000610 \\
\hline I & 2.3578 & 0.000910 & 2.9850 & 0.00003 & 0.1043 \\
\hline J & 2.758 & 0.000635 & 1.065 & 0.179976 & 0.06258 \\
\hline K & 1.0168 & 0.03497 & 1.8697 & 0.00012 & 0.04157 \\
\hline Mean & 1.4993 & 0.04632 & 1.0931 & 0.060032 & 0.040479 \\
\hline
\end{tabular}

using the amount of gaze shifts and the answer time. It means there are still hidden explanation variables. However, the pvalue of the gaze shifts goes below 0.05 . Therefore, the amount of gaze shifts certainly is one of the explanation variables for the edit distances. Note that the $\mathrm{p}$-value and adjusted-R squared for the edit distance calculated from all words are better than those for the edit distance calculated from only placed words. When students answer the sort test of English, they do not stop thinking about words which have already been placed to reduce cognitive load. They would be conscious of all words to find the best combination of them.

In this experiment, we focused on movement students took to put each words. However, it seems students answers are influenced by the flow of sentences. It is expected to improve the cognitive load estimation with consideration of each chunk which is formed based on English grammar.

\section{B. Lack of knowledge revealed with cognitive load}

The result of this study shows that we can estimate the confidence of students from analysis of their behavior. Since the confidence is influenced by the cognitive load, we can estimate the cognitive load by looking behavior of students.

As we discussed in section V.A, when students answer the word sort test of English, they do not stop thinking of words they have already placed to reduce their cognitive load. They keep being conscious of all words, to find the best combination of them. Considering all words is hard work, which arises high cognitive load. Therefore, they try to combine words as chunks. The chunks correspond to confidential parts in their answer. Since each chunk occupies only one working memory, they can decrease the load of memory. Students can store all words in the assignment on the working memory, utilizing chunks. At that time, they can solve it.
Students seem to divide the whole task into small tasks. The process of their card placement appears, according to the order in which the students solve the small tasks. If one small task imposes high cognitive load on a student, the student takes either of a long time to solve it or a miss operation. Our method finds these tasks causing high cognitive load. These tasks tell what kinds of lacks in knowledges students have. Students can reduce the cognitive load when they store appropriate knowledges in their long term memory. On the other hand, if they do not have these knowledges, they have operate lots of information in their working memory. In this case, the cognitive load is high. Therefore, we can find the lack of knowledges.

Improvement of our method would enable teachers to know the lack in knowledge of each student. The method would make it easy for teachers to take care of students in the best way for each.

\section{CONCLUSION}

We proposed a method which contributes to estimating students understanding level. In this method, we analyze behaviors of students in the English word sort assignment in a VR space. The analysis reveals students understanding level from their cognitive load. Students who have low confidence due to their lack of knowledge hesitate about answering the questions. Such students often stop their hands. They also show more gaze shift which come from comparison of their answers with hints. We had an experiment to confirm the relationship of the hands movements with the confidence. We examined the edit distances which represents how many correction is needed to finish English word sort assignment, We confirmed it influences the cognitive load. We also checked the relationship of the gaze shifts with the edit distances. According to the result of the experiment, we can estimate 
the confidence of students from their hands movements of students except those who got poor scores. Moreover, the gaze shifts can be one explanatory variables to explain the edit distances. However, the gaze shifts cannot fully explain the edit distances. We need to find more explanatory variables.

If we can estimate the confidence of students by analyzing the observable value, we can know the cognitive load of students, while they are answering the tests. The cognitive load tells us where their weak points stay, which enables teachers to improve their teaching for each student. Consequently, this method promotes students understanding.

\section{REFERENCES}

[1] Kalyuga, S., Chandler, P., Tuovinen, J., \& Sweller, J. (2001). When problem solving is superior to studying worked examples. Journal of Educational Psychology, 93, 579-588

[2] Schnotz, W., \& Kürschner, C. (2007). A reconsideration of cognitive load theory. Educational Psychology Review, 19(4), 469-508.

[3] Sweller, J., van Merriënboer, J. J. G., \& Paas, F. G. W. C. (1998). Cognitive architecture and instructional design. Educational Psychology Review, 10(3), 251-296.

[4] Miller, G. A. (1956). The magical number seven, plus or minus two: Some limits on our capacity for processing information. Psychological Review, 63, 81-97.

[5] Peterson, L., \& Peterson, M. (1959). Short-term retention of individual verbal items. Journal of Experimental Psychology, 58, 193-198.

[6] Ericsson, K. A., \& Kintsch, W. (1995). Long-term working memory. Psychological Review, 102, 211-245

[7] Sweller, J. (2005). Implications of cognitive load theory for multimedia learning. In R. E. Mayer (Ed.), The Cambridge handbook of multimedia learning (pp. 19-30). New York: Cambridge University Press

[8] Sweller, J., \& Chandler, P. (1994). Why some material is difficult to learn. Cognition and Instruction, 12(3), 185-233.

[9] Sweller, J., van Merriënboer, J. J. G., \& Paas, F. G. W. C. (1998). Cognitive architecture and instructional design. Educational Psychology Review, 10(3), 251-296.
[10] Marcus, N., Cooper, M., \& Sweller, J. (1996). Understanding instructions. Journal of Educational Psychology, 88, 49-63.

[11] Fred Paas , Alexander Renkl \& John Sweller (2003) Cognitive Load Theory and Instructional Design: Recent Developments, Educational Psychologist, 38:1, 1-4, DOI: 10.1207/S15326985EP3801_1

[12] Cowan, N. (2001). The magical number 4 in short-term memory: A reconsideration of mental storage capacity. Behavioral and Brain Sciences, 24, 87-185

[13] S. Tachi, M. Sato, M. Hirose(2010), "Science of Virtual Reality (バー チャルリアリテイ学)”, The virtual reality society of japan

[14] Dutt, Ashish, Maizatul Akmar Ismail, and Tutut Herawan. "A systematic review on educational data mining." IEEE Access 5 (2017): 1599116005.

[15] V. Ivancevic, M. Celikovic, I. Lukovic, "The individual stability of student spatial deployment and its implications", Int. Symp. Comput. Edu. (SIIE), pp. 1-4, Oct. 2012.

[16] K. Nakamura, K. Kakusho, M. Murakami, and M. Minoh(2010). ”Estimating Learners' Subjective Impressions of the Difficulty of Course Materials by Observing Their Faces in e-Learning" The IEICE Transactions on Information and Systems(Japanese Edition) Vol.J93-D No.5 pp.568-578

[17] Richard A. Monty and John W. Senders. 1976. Eye Movements and Psychological Processes. Lawrence Erlbaum Associates, Hillsdale, NJ.

[18] FOVE. FOVE Eye Tracking Virtual Reality Headset. Retrieved September 19, 2017 from https://www.getfove.com/

[19] Mathieu Nancel, Olivier Chapuis, Emmanuel Pietriga, Xing-Dong Yang, Pourang P. Irani, and Michel Beaudouin-Lafon. 2013. High-precision pointing on large wall displays using small handheld devices. In Proceedings of the SIGCHI Conference on Human Factors in Computing Systems - CHI'13, 831. https://doi.org/10.1145/2470654.2470773

[20] Marcos Serrano, Barrett Ens, Xing-Dong Yang, and Pourang Irani. 2015. Gluey: Developing a Head-Worn Display Interface to Unify the Interaction Experience in Distributed Display Environments. In Proceedings of the 17th International Conference on Human-Computer Interaction with Mobile Devices and Services - MobileHCI'15, 161-171. https://doi.org/10.1145/2785830.2785838

[21] Kruger, Justin; Dunning, David (1999). "Unskilled and Unaware of It: How Difficulties in Recognizing One's Own Incompetence Lead to Inflated Self-Assessments". Journal of Personality and Social Psychology 77 (6): 1121-34 